\title{
An Electrochemical Study of POPC Phospholipid Bilayers in a Cell Membrane
}

\author{
A. ELSAGH, K. ZARE and M. MONAJJEMI* \\ Department of Chemistry, Science and Research Branch, Islamic Azad University, Tehran, Iran. \\ *Corresponding author E-mail: m_monajjemi@srbiau.ac.ir \\ http://dx.doi.org/10.13005/ojc/320530 \\ (Received: June 03, 2016; Accepted: August 17, 2016)

\begin{abstract}
In this study, we have shown the model of a microscale dielectric membrane capacitor including POPC structures. We have shown that Quantum effect has appeared in a small free space of the membrane thickness due to number of phospholipids layers. In addition, we have speciûcally investigated the quantum and coulomb blocked effects of diûerent thickness. Furthermore, the electron density profile models, electron localization function (ELF) and local information entropy have been applied as a formal way to studies the interaction of protein with lipid bilayers in cell membrane.
\end{abstract}

Keywords: Variable capacitor, Lipid bilayers, POPC, Cell membrane.

\section{INTRODUCTION}

1-palmitoyl-2-oleoylsn-glycero-3phosphocholine bilayers (POPC) has important rule in the electrochemical properties of a cell membrane in the fluid phase. The composition of a cell membrane directly affects cell membrane functions include membrane permeability, cell signaling, and cell capacitance ${ }^{1-2}$.

Volke and Pampel were the first to report that cross peaks between the lipid 1-palmitoyl-2leolyl-sn-glycero-3-phosphocholine (POPC) and water at a concentration of nine water molecules per POPC have negative intensity, compared to positive intensity for all lipid-lipid cross peaks ${ }^{3}$. The laboratories of Stark confirmed that cross peaks between water and lipid head groups have negative intensity, but the cross peak to the methylene resonance of hydrocarbon chains was weak and positive ${ }^{4}$.

The lipid portion of the membrane serves various barrier functions, preventing most molecules and ions from passing in or out. The membrane covered on both sides by collections of charged dissolved minerals, which serve the same function as a conducting metal plate. 
Because the exterior cell membrane, like the mitochondria in animals and the chloroplasts in plants are biological capacitors they have the capacity to accumulate and store charge and hence energy to be given up when needed. Since energy is needed to run any type of machinery is mechanical or biological it makes sense that nutrients that can enhance energy production and energy storage can have profound biological effects.

Many properties such as cholesterol's impact, heat capacity changes due to increasing salt concentration and correlative lipid motion during diffusion, thickness fluctuations and bilayer undulations have been studied for lipid membrane ${ }^{1-3}$. Local interaction of membrane proteins and lipids as well as the impact of fluorescent probes on bilayer properties has also been investigated by atomistic MD simulations ${ }^{5-9}$.

Except a few causes, it is mostly believed that the lipid bilayer itself is impermeable to, ions, and molecules ${ }^{10}$. Therefore charged molecules must cross through ion channels. Ion channels are transmembrane protein molecules that contain aqueous pores connecting the inside of the cell to the extracellular space. These channels can open and shut in response to a variety of signals. The passage of charged molecules through ion channels in the cell membrane endows the membrane with an electrical conductive property allowing for inward and outward current flows ${ }^{11}$ This is one factor that establishes electric circuits in biological tissues.

So, electrophysiology and dynamic behavior of phospholipids consider the membrane to be a variable capacitor, and ion channel proteins are regarded as electrical resistors. The nerve pulse, for instance, is considered as a propagating segment of charged capacitor loaded by currents through the channel proteins ${ }^{12}$.

The natural properties of membrane structures enable cell components and whole cells to oscillate and interact resonantly with other cells. According to Smith and Best ${ }^{13}$, the cells of the body and cellular components possess the ability to function as electrical resonators.
The outcome of receptor activation may: increase the transport of certain molecules or mineral ions from one side of the cell membrane to the other side; increase or inhibit the activity of enzymes involved in metabolic synthesis or degradation; activate genes to produce certain proteins; turn off gene production of other proteins or cause cytoskeletal proteins to change the shape or motility of the cell. When the receptor protein switches back to its inactive conformation it will detach from the effector proteins/enzymes and the signal wills cease $^{14}$.

The soluble signaling molecules may be produced in distant sites by endocrine cells or are secreted by cells embedded such as macrophages, T-cells and B-cells. When these soluble signaling molecules are presented to the organ cells they can either activate or inhibit cellular metabolic reactions by activating cell membrane or cytoplasmic glycoprotein receptors ${ }^{15}$.

Cell receptors can also be activated by electric fields or vibrational resonance that has particular frequencies and amplitudes through a process known as electro-conformational coupling ${ }^{16}$

Electrical oscillations of the right frequency and amplitude can alter the electrical charge distribution in cell receptors causing the cell receptors to undergo conformational changes just as if the receptor was activated by a chemical signal.

A number of membrane proteins as well as DNA consist of helical coils, which may allow them to electronically function as inductor coils. Because of membrane proteins and DNA function as electrical inductors they may enable the cell to transiently produce very high electrical voltages.

The cell membrane is a leaky dielectric which means that any condition, illness or change in dietary intake that affects the composition of the cell membranes and their associated minerals can affect and alter cellular capacitance.

\section{Theoretical background}

In the case of thin-membrane configurations, this counters the geometric capacitance, ${ }^{C_{m e m}^{g e o}}$ 
$C_{m e m}^{g e o}=\frac{\sigma}{\Delta V_{m e m(t)}}=\frac{s_{r} \varepsilon_{0} \text { jed }}{d_{m e m(t)}}$ voltage, $\begin{array}{r}\Delta V_{m e m}, \text { as } \\ \ldots(1)\end{array}$

where $\delta$ is the surface charge density of membrane, ${ }^{\varepsilon_{0}}$ is the permittivity of free space

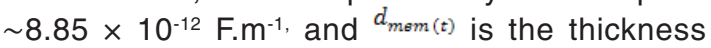
expectation filling by the alkyls chain as a dielectric of the membrane phospholipids. The membrane variable capacitance, ${ }^{C_{m e m}}(t)$, defines how much charge, ${ }^{q}$, is stored on two capacitor plates at a fixed membrane voltage as a function of time, $\left\{V_{\text {mem }}(t)\right\}$. Obviously, quantum electrical characteristics should be considered because of the nano scale property in a membrane cell.

In our model, a small capacitor of cell membrane is made by creating an insulating layer of alkyl between two phosphate groups. We assume that the capacitor electrodes carry $\pm Q$ charges from one electrode towards the opposite side. So the initial energy stored in the electrostatic field between the capacitor plates is given by $E_{i}=\frac{Q^{2}}{2 C}$

By letting the electrons tunnel through the insulating layer from the negative terminal to the positive terminal, such that the charge $\left(Q+\Delta q_{e}\right)$ resides on the top plate and $\left(-\mathrm{Q}-\Delta \mathrm{q}_{\mathrm{e}}\right)$ resides on the bottom plate, the stored energy in this situation is now $E_{f}=\frac{(Q+\Delta q)^{2}}{2 C}$

For small systems, the capacitance may be so trivial that the charging energy " $2 C$ " becomes great and the energy value for tunneling into the quantum system would then increase.

The tunneling resistance ${ }^{17-22}$ can be assumed to be $R_{\text {Tun }}=\frac{\Delta V}{I}$ which not a usual resistance, however, is theoretically allowing electrons to cross the insulating junction as discrete occurrences where "I" is the resulting current due to the tunneling effect. Tunneling resistance is not an usual resistance, but an imaginary one, allowing the electrons to cross the insulating junction during " $\mathrm{t}={ }^{R}$ Tun $C_{Q}$

where $C_{Q}$ is the quantum capacitance and $t$ is a characteristic time associated with tunneling events which is considered to be the approximate lifetime of the energy state of the electrons (on one side of

the barrier). The $R_{T u n}$, should be finite and not too big, so that tunneling can practically take place. In this case, the charge is said to be well quantized and the capacitor is considered to be a tunnel junction in the membrane.

In this work we have calculated the $R_{\text {Tun }}$ for the membrane as a function of the thickness of and difference in potential energy barrier between the electrodes (Via the uncertainty relationship between time and energy,

$$
\Delta E \Delta t \geq \frac{\hbar}{2} \rightarrow R_{\text {Tun }} \geq \frac{h}{2 \pi q_{\varepsilon}^{2}}
$$

The hybrid capacitance and then quantum capacitance are related to the net capacitance, ${ }^{C_{\text {net }}}$ , through the relation $\frac{1}{c_{n e t}}=\frac{1}{c_{g}}+\frac{2}{c_{Q}}$ and the value of $C_{Q}$ is many orders of magnitude greater than the $\mathrm{C}_{\mathrm{g}}$. Hence, its effect usually appears only in quantum systems.

In this work, we show that in the fluid mosaics of lipids the dielectric constant for a membrane has changed time by time while the changing of capacitance is not fast and is depended to the situation of membrane processing. Quantum effect in the lipid membrane implies that the capacitance of the membrane is larger than that of the geometry membrane shows us. Thus, any phenomenon in the bio-membrane related to the dynamic behavior such as physiological temperature will influence its capacitance.

We exhibit the cellular electrical properties and electromagnetic fields effects on cells cases the electro biological phenomenon such as ligand receptor interactions of hormones, alteration of mineral entry through the cell membrane and activation or inhibition of cytoplasmic enzyme reactions.

We show here that the quantum effect is able to change the capacitance membrane due to any external phenomenon. We also show 
how the electrical properties of the membrane are affected by the application of electron density arising from Phosphors in the phospholipids in a membrane. This gives rise to phosphors-induced capacitive currents or phosphors-induced voltage changes. The hybrid and quantum canacitances are related to the net capacitance, ${ }^{C_{m e m}}$, through the relation indicated in equation (2) and scheme 1 : $\frac{1}{c_{m e m}}=\frac{1}{c_{m e m}^{g e o}}+\frac{2}{c_{m e m}^{q u a}}$

Thomas Heimburg and coworkers have shown that the voltage changes during the nerve pulse are actually related to changes in capacitance and a transition voltage is able to change the dimensions of a membrane and the capacitance. They also show how the electrical properties of the membrane are affected by the application of lateral pressure or tension in a membrane ${ }^{23-25}$ furthermore the assumption of constant capacitance is incorrect, especially if one is close to chain melting transitions in the lipid membrane ${ }^{26}$.

In this works we assumed that the $C_{m e m}^{g e o}$ of bio-cell membranes (in contrast of $C_{m e m}^{q u a}$ ) is independent of voltage and only is dependent to geometry so that the second term on the right of this equation is a quantum effect

$\frac{d c_{\text {mem }}^{g e 0}}{d t}=0$

Consider a membrane with variable thickness between $\mathrm{d}_{1}$ and $\mathrm{d}_{2}$, the capacitances will be changed between $\mathrm{C}_{1}={ }^{\varepsilon_{0} \varepsilon \frac{A}{\mathrm{~d} 1}}$ and $\mathrm{C}_{2}={ }^{\varepsilon_{0} \varepsilon \frac{A}{\mathrm{~d} 2}}$ and two transmembrane voltages of $V_{1}$ and $\mathrm{V}_{2}$. The two fields across the membrane are $E_{1}=\frac{V_{1}}{\mathrm{~d} 1}$ (9) and $E_{2}=\frac{V_{2}}{\mathrm{~d} 2}(10)$ and the section of charges is $\sum_{\mathrm{i}=\mathrm{d} 1}^{\mathrm{j}=\mathrm{d} 2} C_{i} V_{i}$.

\section{Electron localization function (ELF)}

The lack of an absolute scale has often greatly hindered the interpretation of membrane profiles, particularly with regard to the distribution of the protein molecules.

In this work it has been described a novel method of the step-function models for the membrane electron density profile in the composition of the lipids. In this work we have focused on the electron density of the systems when the phospholipids have been created one by one in two side of membrane upper lateral side (+) and downer lateral side(-).

$\rho(r)=\eta_{i}\left|\varphi_{i}(r)\right|^{2}=\sum_{i} \eta_{i}\left|\sum_{l} C_{l, i} \chi_{i}(r)\right|^{2}$ ...(11)

Where $\eta_{i}$ is occupation number of orbital $i$, $\varphi$ is orbital wave function, $\mathrm{c}$ is basis function. $C$ is coefficient matrix, the element of $i_{\text {th }}$ row $j_{\text {th }}$ column corresponds to the expansion coefficient of orbital $j$ respect to basis function $i$. Atomic unit for electron density can be explicitly written as e/Bohr ${ }^{3}$.

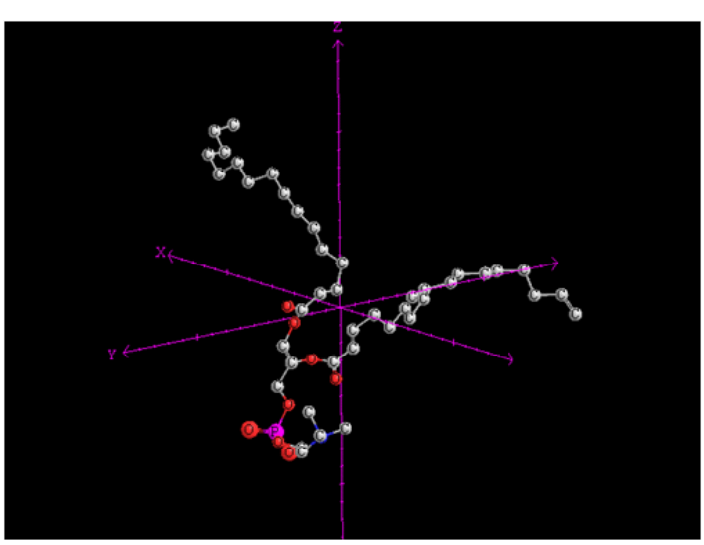

Scheme 2: The POPC molecule including 52 atoms membrane including 120 POPC molecules 
Bader $^{27}$ found that the regions which have large electron localization must have large magnitudes of Fermi-hole integration. However, the Fermi hole is a six-dimension function and thus difficult to be studied visually. Becke and Edgecombe noted that spherically averaged likespin conditional pair probability has direct correlation with the Fermi hole and then suggested electron localization function (ELF) ${ }^{28}$.

$\operatorname{ELF}(r)=\frac{1}{1+\left[D(r) / D_{0}(r)\right]^{2}}$

where

$\mathrm{D}(\mathrm{r})=^{\frac{1}{2}} \sum_{i} \eta_{i}\left|\nabla \varphi_{i}\right|^{2}-\frac{1}{8}\left[\frac{\left.|| \nabla \rho_{\alpha \alpha}\right|^{2}}{\rho_{\alpha}(r)}+\frac{\left.|| \nabla \rho_{\beta}\right|^{2}}{\beta(r)}\right]$

and

$D_{0(r)}=\frac{3}{10}\left(6 \pi^{2}\right)^{\frac{2}{3}}\left[\rho_{\alpha}(r)^{\frac{5}{3}}+\rho_{\beta}(r)^{\frac{5}{3}}\right]$

for close-shell system, since

$\rho_{\alpha}(r)=\rho_{\beta}(r)=\frac{1}{2} \rho, D$ and $D 0$ terms can be

simplified as

$\mathrm{D}(\mathrm{r})=^{\frac{1}{2} \sum_{i} \eta_{i}\left|\nabla \varphi_{i}\right|^{2}-\frac{1}{8}\left[\frac{|\nabla \rho|^{2}}{\rho(r)}\right]}$

and $D_{0(r)}=\frac{3}{10}\left(3 \pi^{2}\right)^{\frac{2}{3}} \rho(r)^{\frac{5}{3}}$

Savin et al. have reinterpreted ELF in the view of kinetic energy ${ }^{29}$, which makes ELF also meaningful for Kohn-Sham DFT wave-function or even postHF wave-function. They indicated that $D(\mathbf{r})$ reveals the excess kinetic energy density caused by Pauli repulsion, while $D 0(\mathbf{r})$ can be considered as Thomas-Fermi kinetic energy density. Since $D 0(\mathbf{r})$ is introduced into ELF as reference, what the ELF reveals is actually a relative localization.

ELF is within the range of $[0,1]$. A large ELF value means that electrons are greatly localized, indicating that there is a covalent bond, a lone pair or inner shells of the atom involved. ELF has been widely used for a wide variety of systems, such as organic and inorganic small molecules, atomic crystals, coordination compounds, clusters, and for different problems, such as the revealing atomic shell structure, classification of chemical bonding, verification of charge-shift bond, studying aromaticity.
Notice that there is a deficiency of ELF, sometimes with $\mathbf{r}$ going beyond from molecular boundary, $D(\mathbf{r})$ decreases faster than $\mathrm{DO}(\mathbf{r})$ and then ELF reaches 1 (completely localized).

To overcome the problem, Multiwfn automatically adds a minimal value $10-5$ to $D(\mathbf{r})$. This treatment almost does not affect the ELF value in interesting regions ${ }^{30}$. In which the actual kinetic energy term in $D(\mathbf{r})$ is replaced by Kirzhnits type second-order gradient expansion, that is $\frac{1}{2} \sum_{i} \eta_{i}\left|\nabla \varphi_{i}\right|^{2} \approx D_{0}(r)+\frac{1}{72} \frac{|\nabla \rho|^{2}}{\rho(r)+\frac{1}{6} \nabla^{2} \rho(r)}(17)$ so that ELF is totally independent from wave-function, and then can be used to analyze electron density from X-ray diffraction data. Of course Tsirelson's ELF can also be used to analyze electron density from quantum chemistry calculation, but is not as good as the ELF defined by Becke owing to the approximation introduced in kinetic energy term; however, qualitative conclusions can still be recovered in general.

Localized orbital locator (LOL) is another function for locating high localization regions likewise ELF, defined by Schmider and Becke in the paper ${ }^{31}$

$$
\operatorname{LOL}(r)=\frac{\tau(r)}{1+\tau(r)}, \text { where }(r)=\frac{D_{0}(r)}{\frac{1}{2} \sum_{i} \eta_{i}\left|\nabla \varphi_{i}\right|^{2}}
$$

$D_{0}(r)$ for spin-polarized system and close-shell system are defined in the same way as in ELF. LOL has similar expression compared to ELF. Actually, the chemically significant regions that highlighted by LOL and ELF are generally qualitative comparable, while Jacobsen pointed out that LOL conveys more decisive and clearer picture than $E^{2} F^{32}$. Obviously LOL can be interpreted in kinetic energy way as for ELF; however LOL can also be interpreted in view of localized orbital. Small (large) LOL value usually appears in boundary (inner) region of localized orbitals because the gradient of orbital wave-function is large (small) in this area.

\section{Computational details}

A part of membrane systems including POPC phospholipids have been modeled via QM/MM simulation using Monte Carlo method. Each system was composed of 120 lipid molecules including water molecules. At finite temperature, clusters have finite vapor pressures, and particular cluster sizes are typically unstable to evaporation. Introducing a 
constraining potential enables one to define clusters of desired sizes.

Each of 120 POPC molecules, were carried out with the lateral dimensions of the simulation cell $\left\{L_{x}\right\},\{L y\}$ and three-dimensional periodic boundary conditions were applied, and the cell length normal to the membrane $\left\{L_{z}\right\}$ was allowed to adjust during the simulation to maintain a constant normal pressure of $1 \mathrm{~atm}$. The pressure was maintained by a variant of the extended system formalism, the Langevin Piston algorithm, which reduces oscillations in the cell parameters. The temperature was maintained at $300 \mathrm{~K}$, well is the body temperature and identical to the relevant experiments. Configurations of individual lipids consistent with a mean field were generated by Monte Carlo (MC) simulation, with field values adjusted to obtain agreement with experimental order parameters.

In this investigation, differences in force field are illustrated by comparing the calculated energy by using force fields AMBER and OPLS. Furthermore HyperChem professional release 7.01 is used for the calculations.

The final parameterization of POPC was computed using self-consistent field calculations in order to find the optimal starting geometry, as well as the partial charges. We employed density functional

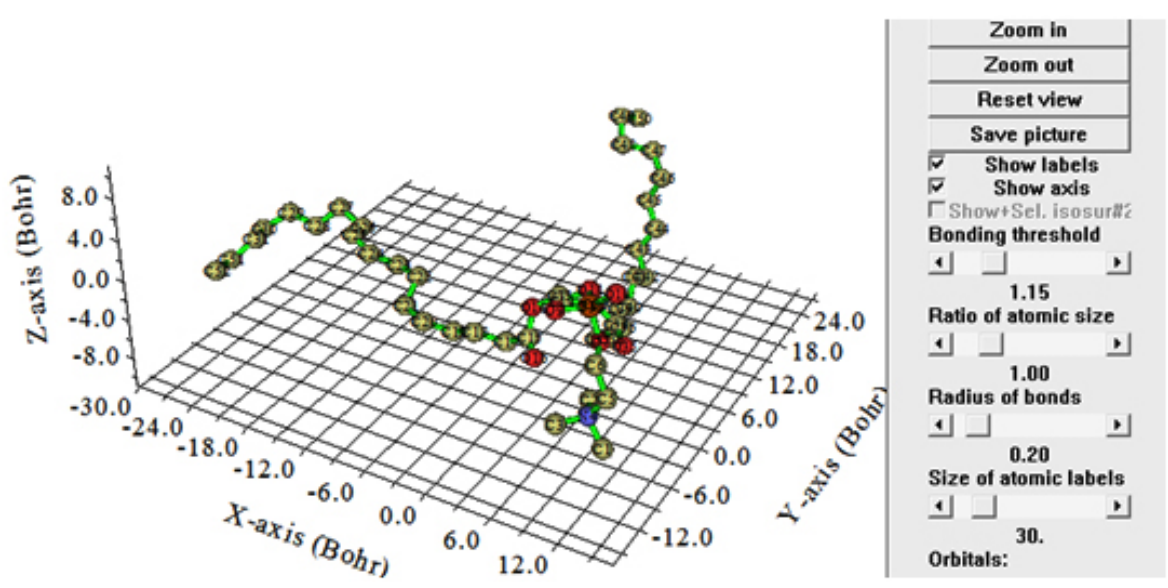

Fig.1: The first molecule optimized of POPC from 120 simulated molecules

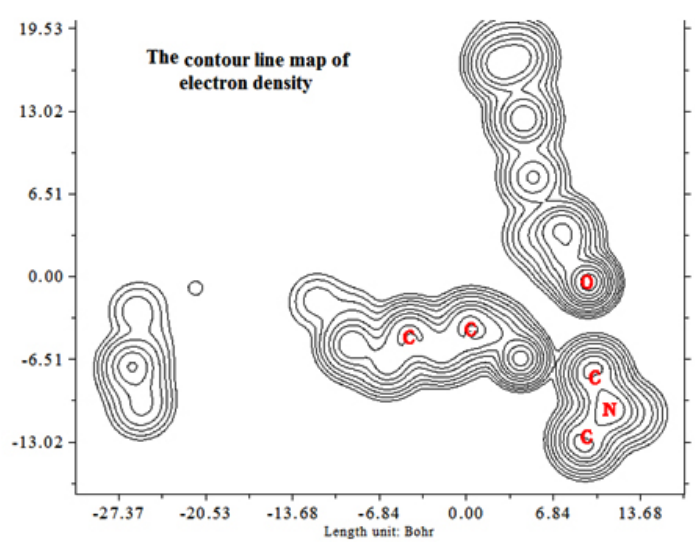

Fig.2: The contour line map of electron density for POPC phospholipids

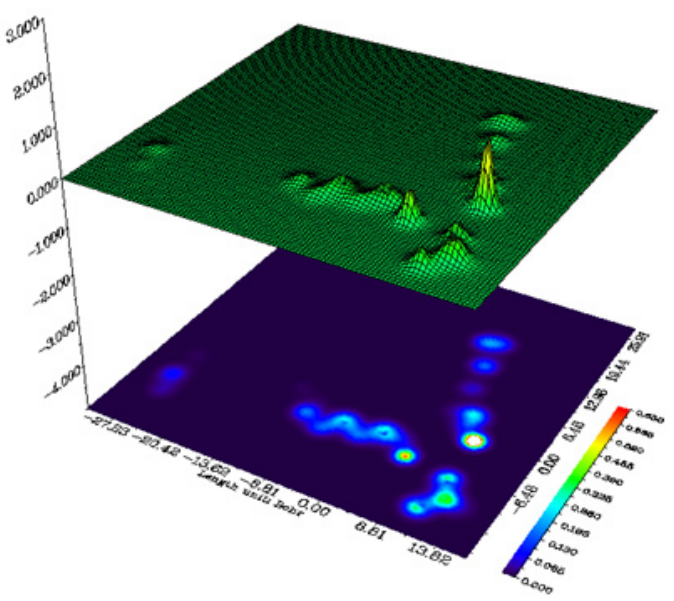

Fig. 3: The electron density with projection for POPC 
theory with the van der Waals density functional to model the exchange-correlation energies of POPC monomers.

All optimization of POPC monomer were performed by Abinitio software. We have mainly focused on getting the results from DFT methods such as m062x, m06-L, and m06 for the (POPC). The m062x, m06-L and m06-HF are rather new DFT functional with a good correspondence in nonbonded calculations between POPC monomers and are useful for the energies of distance between two fragments in phospholipids ${ }^{33}$.

For non-covalent interactions, the B3LYP method is unable to describe van der Waals ${ }^{33,34}$ membrane systems by medium-range interactions such as the interactions of two POPC.

So the ONIOM methods including 3 levels of 1-high calculation $(H)$, 2-medium calculation $(M)$, and 3-low calculation (L) have been performed in

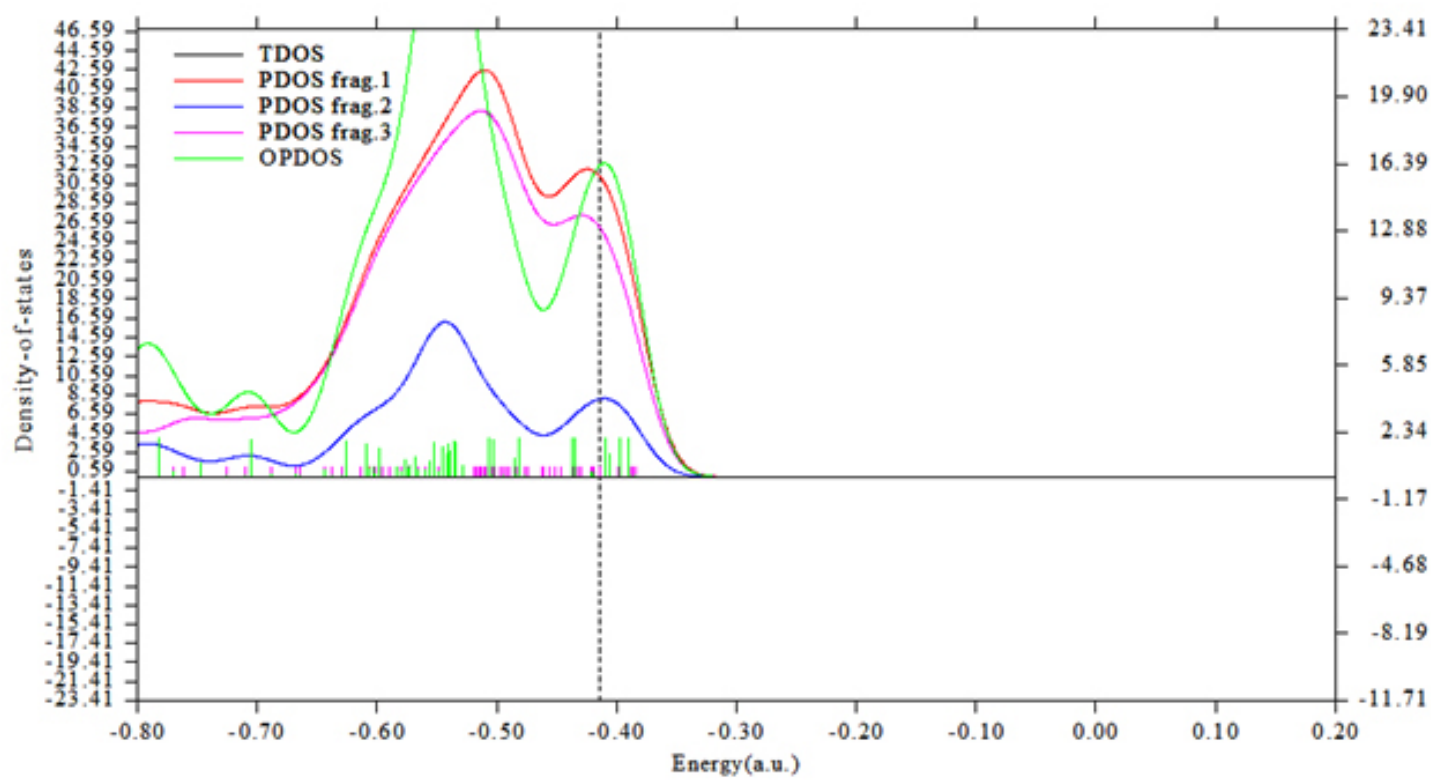

Fig. 4: The Density of state (DOS) including TDOS, PDOS and OPDOS for electrons between two layers of POPC

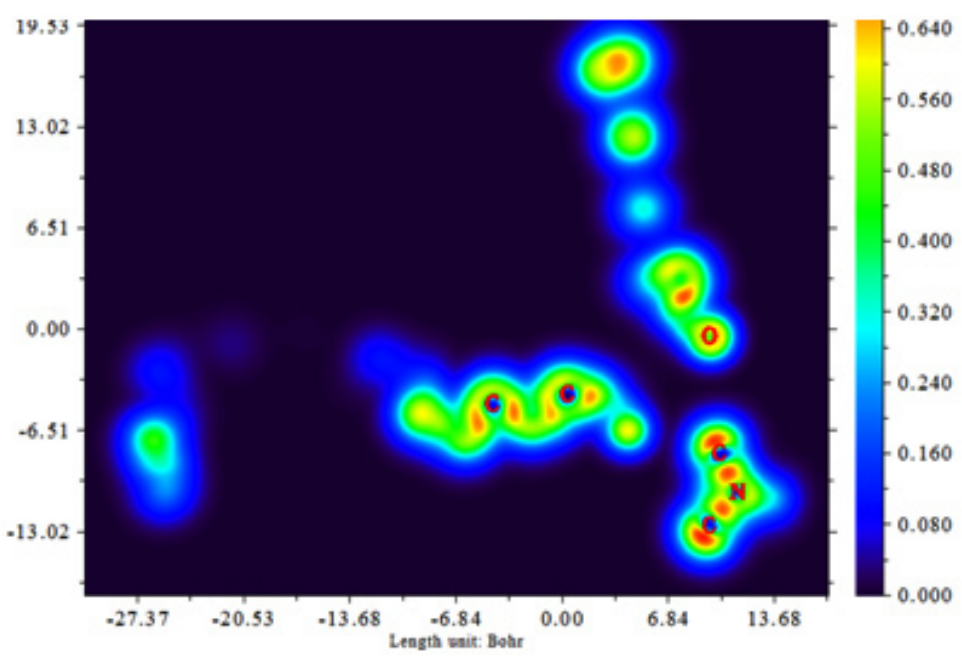

Fig.5: Color field map for the Localized orbital locator (LOL) for POPC 
our study for calculating the non-bonded interactions between phospholipids. The ab-initio and DFT methods are used for the model system of the ONIOM layers and the semi empirical methods of Pm6 (including pseudo=lanl2) and Pm3MM are used for the medium and low layers, respectively. The semi empirical methods have been used in order to treat the non-bonded interactions between two parts of upper lateral phospholipids side $\left({ }^{P_{+}}\right)$and downer lateral phospholipids side $\left({ }^{P_{-}}\right)$.

B3LYP and the most other popular functional are insufficient to illustrate the exchange and correlation energy for distant non-bonded medium-range systems correctly. Moreover, some recent studies have shown that inaccuracy for the medium-range exchange energies leads to large systematic errors in the prediction of molecular properties.

Geometry optimizations and electronic structure calculations have been carried out using the m06 (DFT) functional. This approach is based on an iterative solution of the Kohn-Sham equation ${ }^{35}$ of the density functional theory in a plane-wave set with the projector-augmented wave pseudo-potentials. The Perdew-Burke-Ernzerhof $(\mathrm{PBE})^{36}$ exchange-correlation $(\mathrm{XC})$ functional of the generalized gradient approximation (GGA) is adopted. The optimizations of the lattice constants and the atomic coordinates are made by the minimization of the total energy.

The charge transfer and electrostatic potential-derived charge were also calculated using the Merz-Kollman-Singh ${ }^{37}$, chelp ${ }^{38}$, or chelpG ${ }^{39}$.

The charge calculation methods based on molecular electrostatic potential (MESP) fitting are not well-suited for treating larger systems whereas some of the innermost atoms are located far away from the points at which the MESP is computed.

In such a condition, variations of the innermost atomic charges will not lead towards a significant change of the MESP outside of the molecule, meaning that the accurate values for the innermost atomic charges are not welldetermined by MESP outside the molecule. The representative atomic charges for molecules should be computed as average values over several molecular conformations.

The interaction energy for capacitor was calculated in all items according to the equation 19:

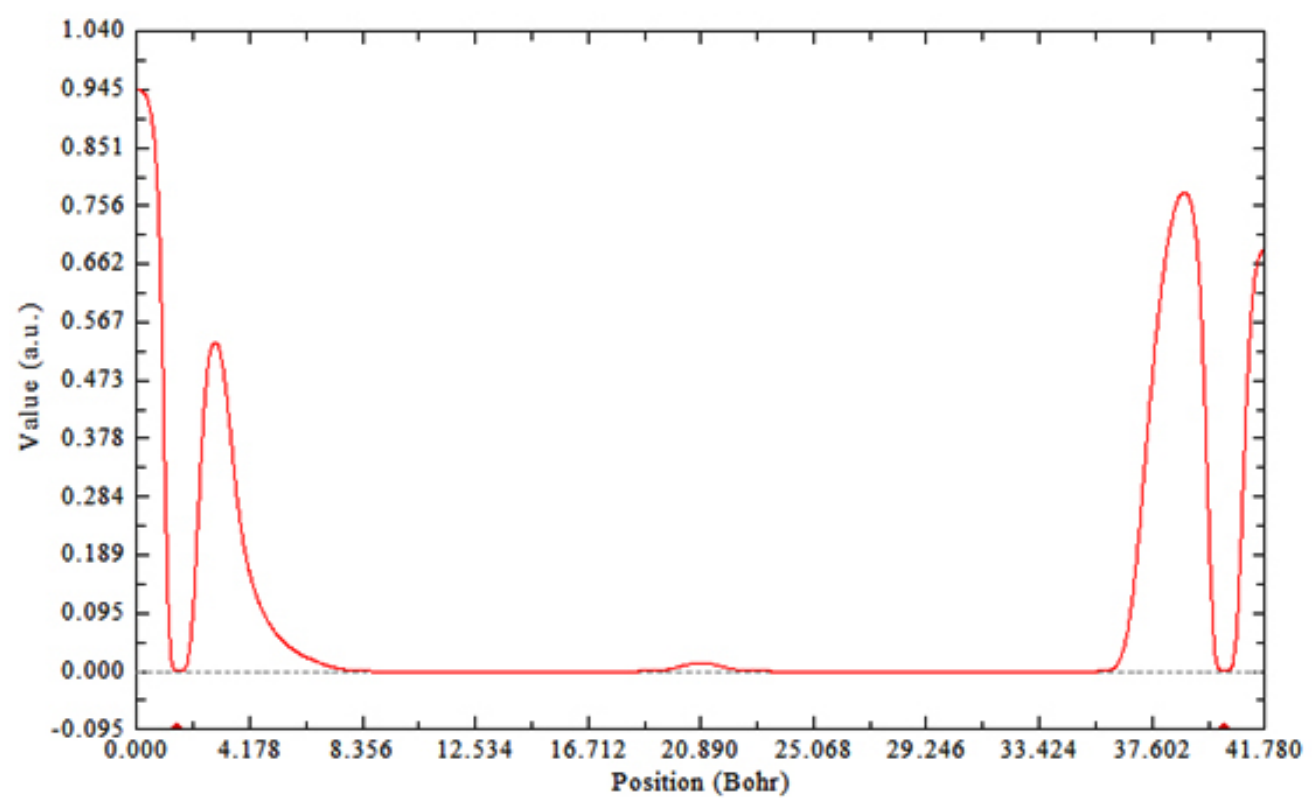

Fig. 6: ELF of between atom numbers 1 to 52 for POPC 


$$
\Delta E_{S}(e V)=\left\{E_{C}-\left(\sum_{i=1}^{40}\left(P O P C_{+}\right)_{i}+\sum_{i=1}^{40}\left(P O P C_{-}\right)_{i}\right)\right\}+E_{B S S E}
$$

Where the " $\Delta E_{S "}$ " is the stability energy of membrane capacitor.

The electron density (Both of Gradient norm \& Laplacian), value of orbital wave-function, electron spin density, electrostatic potential from nuclear atomic charges, electron localization function (ELF), localized orbital locator (LOL defined by Becke \& Tsirelson), total electrostatic potential (ESP), as well as the exchange-correlation density, correlation hole and correlation factor, and the average local ionization energy using the Multifunctional Wavefunction Analyzer have also been calculated in this study $^{40-42}$.

We used Multiwfn software to draw the contour line map ${ }^{41,42}$. The solid lines indicate positive regions, while the dash lines indicate negative regions. We have plotted the contour line corresponding to vdW surface (electron density=0.001 a.u., which is defined by R. F. W Bader). This is useful to analyze distribution of electrostatic potential on vdW surface. Such a contour line has also been plotted in gradient line and vector field map by the same option. Color, label size and line style of the contour line can be changed based on models.

The relief map was used to present the height value at every point. If the values are too large, they will be truncated in the graph. Therefore, it can be chosen to scale the data with a factor to avoid truncation. The graph is shown on interactive interface. Shaded surface map and shaded surface map with projection are used in our representation of height value at each situation ${ }^{40-42}$.

We have applied our previous works ${ }^{43-73}$ and some other methods ${ }^{74-77}$ to exhibit a simple form of biophysical chemistry in a new field of bioelectronics $\leftrightarrow$ biochemistry interactions.

\section{RESULT AND DISCUSSION}

The POPC molecule including 43 atoms is shown in scheme2. It has been shown in the table
1 ; the electrical properties can result from changes in the internal conformation of the lipid as measured by the chain dihedral distributions. Electron density, energy density and Potential energy density for rotation about the ${ }^{\alpha, \beta}$ and $\gamma$ angles were calculated from the angular distributions of each simulation in the Table1 and all data have curved in 6 figures.

Based on eq.19 the larger of electron localization is in a top small region of Fig.1, the more likely the electron motion is confined within it. If electrons are completely localized in the POPC, then they can be distinguished from the ones outside. As shown in table 1 the large ELF is near the point of 163 atoms Fig2. As the regions which have large electron localization must have large magnitudes of Fermi-hole integration, so this phenomena leads the membrane towards upper lateral phospholipids side $\left({ }^{P_{+}}\right)$with exceed electrons and downer lateral phospholipids side $\left({ }^{P_{-}}\right)$with exceed holes Fig.3, however, the fermi hole is a six-dimension function and thus difficult to be studied visually. Based on equations 12, 13 and 14, Becke and Edgecombe noted that spherically averaged like spin conditional pair probability has direct correlation with the fermi hole which it is suitable correspond to our data in table 1 .

ELF reveals is actually a relative localization and must be accounted within the range of $[0,1]$.

A large ELF value means that electrons are greatly localized, indicating that there is a covalent bond, a lone pair or inner shells of the atom involved.

Based on eq.16, LOL can be interpreted in kinetic energy way as for ELF; however LOL can also be interpreted in view of localized orbital. Small (large) LOL value usually appears in boundary (inner) region of localized orbitals because the gradient of orbital wave-function is large (small) in this area. The value range of LOL is identical to ELF, namely $[0,1]$.

LOL has similar expression compared to ELF. Actually, the LOL conveys more decisive and clearer picture than ELF. In table. 1 the LOL is low and constant for both lateral phospholipids sides 
$\left({ }_{ \pm}\right)$(Fig.3). The results of ELF and LOL indicate that the phospholipids membrane starting to become a capacitor in any scales from nano to micro and medium scales.

As shown in the Figure 4, the torsional distributions are independent of the free space between two layers in a dynamic situation this free space is a suitable distance for coulomb blockade and tunneling events. Evidently, as the surface area is increased, the membrane thins sufficiently to maintain essentially the same environment for the alkyl chains (the bilayer interior is similar "but not, clearly" to a liquid alkane in terms of chain conformations).

Expectation of dielectric thickness based on a Gaussian's curve distribution has been calculated as 37.98 for 120 POPC of the membrane. Electron density profiles, displayed in Figure 5, were calculated from the electron density profile models by dividing the simulation cells into $0.1 \AA$ slabs and determining the time-averaged number of electrons in each slab. The peaks show the position of the electron-rich phosphate section of the head group and the peak-to-peak distance $(\mathrm{XHH})$ is often used

Table 1: Electron Density, Energy Density H(r), Electron localization Function (ELF), Localized Orbital Locator (LOL), from nuclear charge and from electron charge for Phosphor in POPC

\begin{tabular}{|c|c|c|c|c|c|c|c|c|}
\hline $\begin{array}{l}\text { POPC\& } \\
\text { Number } \\
\text { of atoms }\end{array}$ & $\begin{array}{l}\Delta \mathrm{Es} \\
(e V)\end{array}$ & $\begin{array}{l}\text { ALIE* }^{*} \\
\text { LIE }\end{array}$ & & $\begin{array}{l}\text { ELF}^{\star} \\
\text { LOL }^{\dagger} \\
\text { ESp\# }\end{array}$ & & $\begin{array}{l}\alpha^{\circ} \pm n \\
\beta^{\circ} \pm n \\
\gamma^{\circ} \pm k\end{array}$ & & $\begin{array}{l}\text { Electron Density* } \\
\text { a.u.Energy Density } \\
H(r)^{\dagger}(e V)\end{array}$ \\
\hline $1_{+}(\mathrm{N}=52)$ & 0.0 & & $\mathrm{P}_{1+}$ & $\begin{array}{l}1.25(E-04)^{*} \\
1.11(E-02)^{\dagger} \\
2.43(E+01)^{\#}\end{array}$ & $P_{1+}$ & $\begin{array}{l}111.2^{\circ} \\
114.5^{\circ} \\
112.3^{\circ}\end{array}$ & $\mathrm{P}_{1+}$ & $\begin{array}{l}4.19(\mathrm{E}-03)^{*} \\
3.99(\mathrm{E}-01)^{\dagger}\end{array}$ \\
\hline $\begin{array}{l}1,1 \\
(\mathrm{~N}=104)\end{array}$ & +1.44 & $\begin{array}{l}P_{1+}=-0.06 \\
P_{1-}=-0.07\end{array}$ & $P_{1+}: P_{1-}$ & $\begin{array}{l}\text { 1.35: } 2.06(E-04)^{*} \\
1.45: 2.11(E-02)^{\dagger} \\
-4.6:-5.2(E+01)^{\#}\end{array}$ & $P_{1+}: P_{1-}$ & $\begin{array}{l}\text { 110.0:111.3 } \\
\text { 112.2:112.5 } \\
113.5: 111.6\end{array}$ & $P_{1+}: P_{1 .}$ & $\begin{array}{l}4.29: .4 .37(\mathrm{E}-03)^{*} \\
4.86: 4.26(\mathrm{E}-01)^{\dagger}\end{array}$ \\
\hline $1,1$. & & & $P_{1+}: P_{1-}$ & $\begin{array}{l}\text { 1.35: } 1.46(E-04)^{*} \\
1.05: 1.14(E-02)^{\dagger}\end{array}$ & $\mathrm{P}_{1+}: \mathrm{P} 1$ & $\begin{array}{l}\text { 111.0: } 112.1 \\
\text { 112.3: } 112.8\end{array}$ & $P_{1+}: P_{1-}$ & $\begin{array}{l}4.29: .4 .45(E-03)^{*} \\
3.91: 4.26(E-01)^{\dagger}\end{array}$ \\
\hline $\begin{array}{l}2,2 \\
(\mathrm{~N}=156)\end{array}$ & +1.87 & & $P_{2+}: P_{2-}$ & $\begin{array}{c}-4.1:-7.3(E+01)^{\#} \\
\text { 1.29:1.31 (E-04) } \\
\text { 1.12:1.28) E-02) } \\
-4.13:-6.90(E+01)^{\dagger}\end{array}$ & $P_{2+}: P_{2-}$ & $\begin{array}{l}113.7: 111.4 \\
114.0: 113.6 \\
112.5: 111.3 \\
112.1: 111.9\end{array}$ & $\mathrm{P}_{2+}: \mathrm{P}_{2-}$ & $\begin{array}{l}\text { 4.19: } 4.25(E-03)^{*} \\
3.11: 4.28(E-01)^{\dagger}\end{array}$ \\
\hline $\begin{array}{l}1_{+}, 1 \\
2,2 \\
3_{+}, 3\end{array}$ & +0.9 & $\begin{array}{l}P_{1+}=-0.07 \\
P_{1-}=-0.08 \\
P_{2+}=-0.09\end{array}$ & $P_{1+}: P_{1-}$ & $\begin{array}{l}\text { 1.25: } 1.36(\mathrm{E}-04)^{*} \\
\text { 1.25: } 1.21(\mathrm{E}-02)^{\dagger} \\
-4.13:-6.9(\mathrm{E}+01)^{\#}\end{array}$ & $P_{1+}: P_{1-}$ & $\begin{array}{l}\text { 112.1:113.4 } \\
\text { 112.3:111.5 } \\
111.6: 112.9\end{array}$ & $P_{1+}: P_{1-}$ & $\begin{array}{l}\text { 4.19:.4.10 (E-03) } \\
4.38: 4.28(E-01)^{\dagger}\end{array}$ \\
\hline \multirow[t]{4}{*}{$\begin{array}{l}4_{+}, 4 \\
5_{+}, 5 \\
(\mathrm{~N}=520)\end{array}$} & & $P_{2}=-0.06$ & $\mathrm{P}_{2+}: \mathrm{P}_{2-}$ & $\begin{array}{c}1.39: 1.41(E-04)^{*} \\
1.12: 1.28) E-02)^{\dagger} \\
-4.3:-7.20(E+01)^{\#}\end{array}$ & $P_{2+}: P_{2-}$ & $\begin{array}{l}\text { 111.0: } 111.2 \\
112.1: 111.5 \\
111.6: 110.9\end{array}$ & $P_{2+}: P_{2-}$ & $\begin{array}{l}\text { 3.23: } 4.16(E-03)^{*} \\
\text { 4.25: } 4.38(E-01)^{\dagger}\end{array}$ \\
\hline & & $\begin{array}{l}P_{3+}=-0.05 \\
P_{3-}=-0.07\end{array}$ & $P_{3+}: P_{3-}$ & $\begin{array}{c}1.17: 1.25(E-04)^{*} \\
1.13: 1.03) E-02)^{\dagger} \\
-4.28:-7.4(E+01)^{\#}\end{array}$ & $P_{3+}: P_{3-}$ & $\begin{array}{l}\text { 112.1:113.2 } \\
\text { 110.2:112.2 } \\
\text { 111.5:110.3 }\end{array}$ & $P_{3+}: P_{3-}$ & $\begin{array}{l}\text { 3.19: } 3.10(E-03)^{*} \\
4.02: 4.25(E-01)^{\dagger}\end{array}$ \\
\hline & & $P_{4+}=-0.03$ & $\mathrm{P}_{4+}: \mathrm{P}_{4-}$ & $\begin{array}{c}1.16: 1.25(E-04)^{*} \\
1.32: 1.18) E-02)^{\dagger} \\
-3.21:-5.35(E+01)^{\#}\end{array}$ & $\mathrm{P}_{4+}: \mathrm{P}_{4-}$ & $\begin{array}{l}112.3: 113.2 \\
110.1: 112.5 \\
111.2: 110.8\end{array}$ & $\mathrm{P}_{4+}: \mathrm{P}_{4-}$ & $\begin{array}{l}\text { 3.17:3.26 }(\mathrm{E}-03)^{\star} \\
4.32: 4.31(\mathrm{E}-01)^{\dagger}\end{array}$ \\
\hline & & $P_{4-}=-0.02$ & $P_{5+}: P_{5-}$ & $\begin{array}{l}1.16: 1.25(E-04)^{*} \\
1.26: 1.17)(E-02)^{\dagger} \\
-4.1:-5.6(E+01)^{\#}\end{array}$ & $P_{5+}: P_{5-}$ & $\begin{array}{l}113.1: 112.1 \\
111.3: 112.8 \\
110.4: 111.5\end{array}$ & $P_{5+}: P_{5-}$ & $\begin{array}{l}\text { 3.36: } 3.16(\mathrm{E}-03)^{*} \\
\text { 4.49: } 4.31(\mathrm{E}-01)^{\dagger}\end{array}$ \\
\hline
\end{tabular}


Table 2: Dielectric constant and capacitance of various modeled membrane capacitors

\begin{tabular}{|c|c|c|c|c|c|}
\hline $\begin{array}{l}\text { POPC } \\
\text { membrane } \\
\& \text { Number } \\
\text { of subunits }\end{array}$ & $\begin{array}{l}\text { LUMO/HOMO } \\
\text { (Orbital Number) }^{*} \\
\text { Gap KJ/mol** }^{\star *}\end{array}$ & $\begin{array}{c}\Delta V= \\
\Delta\left(\sum V_{P_{+}}^{1 \operatorname{to} N}\right. \\
\left.-\sum V_{P_{-}}^{1 \text { to } N}\right)\end{array}$ & $\begin{array}{c}\Delta Q= \\
\Delta\left(\sum Q_{P_{+}^{1}}^{1-N}\right. \\
\left.-\left|\sum Q_{P_{-}}^{1-N}\right|\right)\end{array}$ & $C_{g}(F) \times 10^{20}$ & $\begin{array}{l}\text { Dielectric } \\
\text { Constant }\end{array}$ \\
\hline$(\mathrm{N}=1)$ & 2.66 & - & - & - & - \\
\hline$(\mathrm{N}=2)$ & 3.22 & 2.44 & 0.99 & 3.8 & 2.13 \\
\hline$(\mathrm{N}=4)$ & 3.12 & 4.12 & 1.14 & 4.7 & 3.15 \\
\hline$(\mathrm{N}=8)$ & 3.18 & 3.88 & 1.34 & 5.11 & 2.28 \\
\hline
\end{tabular}

Table 3: The capacitance of modeled capacitors in various charges

\begin{tabular}{ccccc}
\hline & \multicolumn{2}{c}{$C_{Q}(F) \times 10^{19}$} & \multicolumn{2}{c}{$C_{n z t}(F) \times 10^{19}=$} \\
& ESP & Mulliken & ESP & Mulliken \\
\hline$(\mathrm{N}=2)$ & 1.43 & 1.98 & 1.55 & 1.48 \\
$(\mathrm{~N}=4)$ & 1.65 & 1.38 & 1.96 & 1.34 \\
$(\mathrm{~N}=8)$ & 1.42 & 1.13 & 1.17 & 1.41 \\
\hline
\end{tabular}

to infer the molecular length or changes in length from the experimental

In this study, POPC was chosen as a membrane capacitor since the alkyl groups is an excellent spacer close to that of biological phenomenon. Since the alkyl chain in membrane cell is an ideal electrical insulator that can be polarized by applying an external electric field, the expectation thickness of alkyl layers between the membrane plates (which has a dynamic behavior) has been calculated and optimized as a suitable simulation of dielectrics for capacitance calculation Table 2.

Similar to other capacitors, the anisotropic binding of alkyl allows for the formation of various layered structures. Long-range interlayer interactions play a dominant role in characterizing the structural and mechanical properties of these systems and hence their performance in the simulation of our capacitor models.

As shown in Fig.5\&6 there are fluctuation within a decreasing amount for electron density, energy density, ELF and LOL while there is no fluctuation for ESP and it is periodic. In The ESP
Curve versus number of Phospholipids a minimum has been occurred in the curve corresponds on add numbers while for the even numbers there are similar values which indicates that the variable capacitance in a membrane is independent of number of phospholipids .in other words the external phenomenon of the cells makes the variable capacitance, not the quantity of internal structures such as number of phospholipids.

The values of the distances between two phospholipid layers, dielectric constants of the layered alkyl (k), difference magnitude of the charges on the plates, electrostatic properties using the SCF density, fitting point charges to electrostatic potential charges from ESP fit, the stability energy of capacitor $(\mathrm{eV})$, various thickness including the net capacitance and the potential difference between two electrodes of membrane plates (upper and downer lateral phospholipids side) are listed in Tables 2, 3.

The potential energy difference between the two layers, $\Delta V=\Delta\left(\sum V_{P_{+}}^{1-N}-\sum V_{P_{-}}^{1-N}\right)($ a.u. $)$ are depicted in table2 and varies between 3.11 and 14.75 volts for various thickness and number of 
phospholipids, which leads to the accumulation of approximately identical amount of surface charges of the opposite sign.

Here we have considered the interlayer attraction using $\mathrm{OM} / \mathrm{MM}$ with an Oniom methods including OPLS (CHARMM), force field and Extended-Huckel for specific layers to describe its interlayer interactions including inter-layer potential, attractive components and the classical mono-polar electrostatic terms.

In this study, via the number of alkyls and volume of membrane, the expectation of area for the upper and downer lateral phospholipids side ()(pales), have been calculated as $A=$ which is between 100-1000 range is changed.

The nano-capacitances of, and for various number of POPC in different thickness of dielectric are listed in table3.

Although the dielectric strength can be deduced from the band gap of alkyl spacer, here we have calculated the dielectric constant straightaway from eq. 5 that is much more accurate than the other methods.
It is debatable that for alkyl spacer containing a few layers, the different voltages between two electrodes would depend on various conditions such as non-equilibrium state and dynamic situation of the coupling between each POPC and adjacent POPC, etc. It however requires a thorough analysis.

The electric fields induce dipole movements which is a function of polarization processes and the strength of the electric field. When biological tissue is exposed to an electric field in the right frequency and amplitude windows a preferential alignment of dipoles becomes established. Bio-membranes are thin capacitors with the unique feature of biological energy storage in a physiologically relevant phenomenon. Resonance communication mechanisms are depended to interaction between membrane capacitors and helical proteins. When high frequency fields are applied with external signal generators this results in charging of the cell membranes causing an increase in cell membrane capacitance and increased conduction of current through the cell membranes. Because cell membranes naturally have capacitance this makes the cell membrane frequency-dependent conductors.

\section{REFERENCES}

1. Roark, M.; Feller, S.E. J. Phys. Chem. B, 2009, 113, 13229-13234.

2. Lindahl, E.; Edholm, O. Biophys. J. 2000, 79, 426-433.

3. Volke, F; Pampel, A, Biophys J, 1995 68:1960-1965

4. Zhou, Z; Sayer, BG; Hughes, DW; Stark, RE; Epand, RM;. Biophys j, 1999, 76:387-399

5. Hub, J.S.; Aponte-Santamaría, C.; Grubmüller, H.; de Groot, B.L. Biophys. J. 2010, 99, L97L99.

6. Pöyry, S.; Cramariuc, O.; Postila, P.A.; Kaszuba, K.; Sarewicz, M.; Osyczka, A.; Vattulainen, I.;Róg, T. Biochim. Biophys. Acta 2013, 1827, 769-778.

7. Hölttä-Vuori, M.; Uronen, R.L.; Repakova, J.; Salonen, E.; Vattulainen, I.; Panula, P.; Li, Z.; Bittman, R.; Ikonen, E. BODIPY-cholesterol: Traffic 2008, 9, 1839-1849.
8. Repáková, J.; Holopainen, J.M.; Karttunen, M.; Vattulainen, I. J. Phys. Chem. B 2006, 110, 15403-15410.

9. Vanselow, K. Med. Biol. Elektronik 1996 11:1-5.

10. Heimburg, T. Biophys. Chem. 2010, 150:222.

11. Aidley DJ, Stanfield PR. Ion Channels: Molecules in Action.1996 Cambridge, UK: Cambridge University Press.

12. Hodgkin, A. L.; A. F. Huxley. J. Physiol 1952. 117:500-544.

13. Smith C, Best S. Electromagnetic Man. New York: St. Martin's Press, 1989.

14. Van Winkle LJ. Biomembrane Transport. San Diego, CA: Academic Press, 1995

15. Reichart. L.F, Oxford, England: Oxford University Press, 1999, 335-344.

16. Tsong TY. Trends in Biochemical Sciences 
1989, 14, 89-92.

17. Averin, D. V.; Likharev, K. K. in Mesoscopic Phenomena in Solids, edited by B. L. Altshuler, P. A.

18. Andres, R. P. ; Bein, T. ; Dorogi, M. Science 1996, 272, 1323 .

19. Soldatov, E. S. ; Khanin, V. V. ; Trifonov, A. S. ; Presnov, D. E. ; Yakovenko, S. A. ; Khomutov, G. B. ; Gubin, G. P. ; Kolesov, V. V. JETP Lett. 1996, 64, 556 .

20. Park, K.H.; Ha, J. S. ; Yun, W. S. ; Shin, M.; Park, K.W. ; Lee, E.H. Appl. Phys. Lett.1997, 71, 1469 .

21. Klein, D. L. ; McEuen, P. L. ; Bowen Katari, J. E. ; Roth, R. ; Alivisatos, A. P. Appl. Phys. Lett.1996, 68, 2574.

22. Ralph, D. C. ; Black, C. T. Tinkham, M. Phys. Rev. Lett.1996, 76, 688.

23. Heimburg, T.; Jackson, A. D. Proc. Natl. Acad. Sci. USA.2005, 102, 9790-9795.

24. Heimburg, T.; Jackson. A. D. Biophys. Rev. Lett.2007, 2:57-78.

25. Lautrup, B.; Appali, R. .; Heimburg, T. Eur. Phys. J E Soft Matter. 2011, 34:1-9.

26. Thomas Heimburg, Biophysical Journal Volume 103 September 2012 918-929

27. Bader, R.F.W. atoms in Molecule: A quantum Theory (Oxford Univ. press, Oxford, 1990.

28. Becke and Edgecombe J. Chem. Phys., 92, 5397

29. Savin et al Angew. Chem. Int. Ed.Engl., 31, 187 ,

30. Tsirelson and Stash, Chem. Phys. Lett., 351, 142

31. Schmider and Becke, J. Mol. Struct. (THEOCHEM), 527, 51

32. Jacobsen Can. J. Chem., 86, 695

33. Zhao, Y.; Truhlar, DG, Theor Chem Account 2008, 120, 215-241.

34. Zhao, Y.; Truhlar DG Theor Chem Account 2008, 41, 2, 157-167.

35. Kohn, W.; Sham, L.J. Phys. Rev 1995, 140 A: 1133-1138.

36. Perdew, J. P.; Burke, K.; Ernzerhof ,Phys. Rev. Lett.1996, (77): 3865-3868.

37. Besler, B.H.; Merz, K.M.; Kollman, P.A J. comp. Chem , 1990, (11): 431-439.

38. Chirlian, L.E.; Francl, M.M , J.comp.chem 1987, 8: 894-905.

39. Brneman, G.M.; Wiberg, K.B , J. Comp Chem
, 1990, (11): 361.

40. Lu, T.; Chen, F, Acta Chim. Sinica, 2011, 69, 2393-2406.

41. Lu, T.; Chen, F, J. Mol. Graph. Model, 2012, (38): 314-323.

42. Lu, T.; Chen, F , J. Comp. Chem. 2012, (33) 580-592

43. Monajjemi, M.; Lee, V.S.; Khaleghian, M.; B. Honarparvar, B.; F. Mollaamin, F. J. Phys. Chem C. 2010, 114, 15315

44. Monajjemi, M. Struct Chem. 2012, 23,551580

45. Monajjemi, M.; Boggs, J.E. J. Phys. Chem. A, 2013, 117, 1670 "1684

46. Mollaamin, F.; Monajjemi, M, Journal of Computational and Theoretical Nanoscience. 2012, 9 (4) 597-601

47. Monajjemi, M.; Khaleghian, M, Journal of Cluster Science. 2011, 22 (4), 673-692; 318

48. Monajjemi, M. Chemical Physics. 2013, 425, 29-45

49. Monajjemi, M.; Wayne Jr, Robert. Boggs, J.E. Chemical Physics. 2014, 433, 1-11

50. Monajjemi, M.; Mollaamin, F. Journal of Computational and Theoretical Nanoscience, 2012, 9 (12) 2208-2214

51. Monajjemi, M. Falahati, M.; Mollaamin, F.; lonics, 2013, 19, 155-164

52. Monajjemi, M.; Mollaamin, F. Journal of Cluster Science, 2012, 23(2), 259-272

53. Tahan, A.; Monajjemi, M. Acta Biotheor, 2011, 59, 291-312

54. Lee, V.S.; Nimmanpipug, P.; Mollaamin, F.; Kungwan, N.; Thanasanvorakun, S.; Monajjemi, M. Russian Journal of Physical Chemistry A, 2009, 83, 13, 2288-2296

55. Mollaamin, F.; Monajjemi, M. Physics and Chemistry of Liquids .2012, 50, 5, 2012, 596-604

56. Monajjemi, M.; Khosravi, M.; Honarparvar, B.; Mollaamin, F.; International Journal of Quantum Chemistry, 2011, 111, 27712777

57. Monajjemi, M., Mahdavian, L., Mollaamin, F. Bull. Chem. Soc. Ethiop. 2008, 22(2), 277286

58. Monajjemi, M.; Farahani, N.; Mollaamin, F. Physics and Chemistry of Liquids, 2012, 50(2) 161-172

59. Monajjemi, M. Theor Chem Acc, 2015, 134:77 
DOI 10.1007/s00214-015-1668-9

60. Monajjemi, M. Journal of Molecular Modeling, 2014, 20, 2507

61. Monajjemi, M.; Khaleghian, M.; Mollaamin, F. Molecular Simulation. 2010, 36, 11, 865-

62. Ilkhani, Ali R.; Monajjemi, M. Computational and Theoretical Chemistry.2015 1074, 19-25

63. Monajjemi, M. Biophysical Chemistry. 2015 $207,114-127$

64. Jalilian,H.; Monajjemi, M. Japanese Journal of Applied Physics. 2015, 54, 8, 08510

65. Mahdavian, L.; Monajjemi, M. Microelectronics Journal. 2010, 41(2-3), 142-149

66. Tahan, A.; Mollaamin, F.; Monajjemi, M. Russian Journal of Physical Chemistry $A$, 2009, 83 (4), 587-597

67. Mehdizadeh Barforushi,M.; Safari,S.; Monajjemi,M.; J. Comput. Theor. Nanosci. 2015, 12, 3058-3065.

68. Rahmati,H.; Monajjemi,M.; J. Comput. Theor. Nanosci.2015, 12, 3473-3481.

69. Tarlani Bashiz,R.; Monajjemi,M.; J. Comput.
Theor. Nanosci.2015, 12, 3808-3816.

70. Mehrabi Nejad,A.; Monajjemi,M.; J. Comput. Theor. Nanosci. 2015, 12, 3902-3910.

71. Monajjemi,M.; Bagheri,S.; Moosavi,M.S..; Moradiyeh,N.; Zakeri,M.; Attarikhasraghi,N.; Saghayimarouf, N.; Niyatzadeh,G.; Shekarkhand,M.; Mohammad S. Khalilimofrad, Ahmadin,H.; Ahadi,M.; Molecules 2015, 20, 21636-21657;

72. Monajjemi,M.; Nayyer T. Mohammadian J. Comput. Theor. Nanosci. 2015, 12, 48954914.

73. Monajjemi, M., Chahkandi, B. Journal of Molecular Structure: THEOCHEM, 2005, 714 (1), 28, 43-60.

74. Naghsh,F, Orient J Chem, 2015, 31(1) 465478

75. Chitsazan, A, Orient J Chem, 2015, 31(1) 393- 408

76. Barmaki, Z, Orient J Chem, 2015, 31(3) 17231733

77. Bonsakhteh, B.; Rustaiyan, A.H, Orient $J$ Chem, 2014, 30(4) 1703-1718. 